\title{
Moving targets and experiments: One publisher's mobile journey
}

\author{
Jenni Rankin \\ Annual Reviews, 4139 El Camino Way, Palo Alto, CA 94306, USA \\ Tel.: +16508436634 \\ Fax: +16504240910 \\ Email: jrankin@annualreviews.org
}

\begin{abstract}
For 80 years, Annual Reviews has been synthesizing the literature to help scientists keep up with the wealth of scientific research that is published each year. Our content has traditionally been delivered online and in print as long-form review articles, but with scientists more pressured than ever, we have looked into mobile platforms for new ways to fit information into readers' workflows. We asked ourselves, How are our users changing? How do their needs or habits differ? What is important to them? By leveraging our core role and strengths, Annual Reviews strives to be innovative in our approach to solving readers' future problems. This article looks at our product development and innovation processes and experiences, with examples of lessons learned and hints on where we are going next.
\end{abstract}

Keywords: Mobile, information, information overload, critical review, workflow, smartphone, tablet, product development, NFAIS

\section{Drinking from the fire hose}

Keeping up with the literature in any given field is like trying to drink from the proverbial fire hose. There is simply too much to take in, and academics frequently report that they feel they are drowning in new knowledge. And yet it is vital to the progress of science and research that scholars are able to keep abreast of new findings. Of course, this is not a new problem. In the early 1930s, Stanford Professor of Biochemistry, Dr. J. Murray Luck, struggled with this issue in his then-emerging field. While attempting to review the already overwhelming literature, he found himself "dismayed ... by the immensity of the task" [1]. He arranged for the leading professors in the field to write intelligent syntheses of the key publications. As a result, the model for all Annual Reviews journals was formulated. Volume 1 of the Annual Review of Biochemistry, which contained 724 pages and included authors from nine countries, came off the press in July 1932. Eighty years later, the organization Dr. Luck founded publishes reviews in more than 40 fields, and continues to be a valuable tool for scholars seeking to understand a new field. Our core products are long-form critical review articles, available online, via mobile device and in print (Fig. 1), that draw together and analyze the significant primary research in a field or topic from an expert's perspective.

\section{The whole is greater than the sum of its parts}

One common sentiment from our readers is that Annual Reviews gives them permission not to read everything. Our experts help them navigate the wider pool of literature by pointing to the most important 


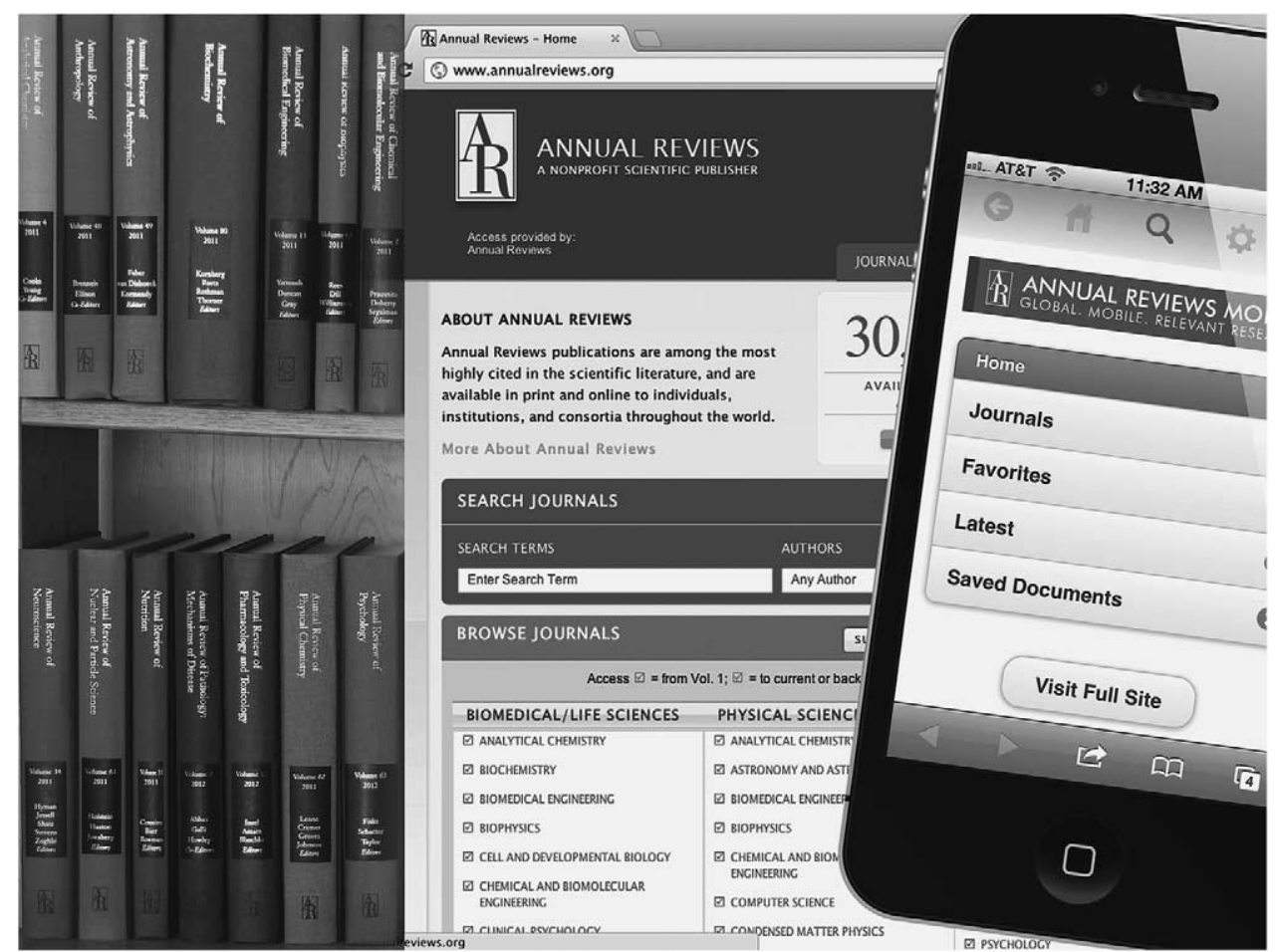

Fig. 1. Annual Reviews has evolved through the information ages.

citations - although around 150 per article, this is a considerable reduction on the complete literature in a field. We're valued as a time-saver, and it is in the context of that core strength that we continue to evolve our products to meet changing user needs. We are conscious that sociological and technological shifts affect our readership, and that the next-generation's academics are Google-centric multi-taskers. We have undertaken research over the past several years to explore how the needs of this group differ from their predecessors and to understand what is important to them. Around 100 early-career researchers, typically between the ages of 25 and 35, have participated in our focus groups and reinforced to us that the critical review is still necessary and valuable; they do not want us to change the essential nature of what we do, but they do want new and convenient formats and content delivery.

\section{Learning and leading}

The pace of change in the mobile environment is such that readers are not yet clear what they want in terms of devices, content or functionality. Our research shows that late twentieth century technology still reigns in the minds of our users: they consider laptops, email, PDFs and table of contents alerts as key tools for finding and consuming content. However, it is quickly becoming apparent that mobile is and will increasingly be a key channel for delivering academic content. For Annual Reviews, mobile's "wherever, whenever" capabilities align well with our role of helping academics keep on top of the literature by helping us fit our content around their workflows. The trends are clear; three years ago, none of our focus group participants owned a small form factor device, but within two years all participants did (a recent split was about 50\% Apple iOS, 50\% Android). Similarly, one year ago, none of our focus 
group participants had a tablet computer. Our recent statistics also show that mobile usage is growing quickly, in line with device adoption. Now, 39\% of our mobile traffic is from the iPad. Users now want content - including images - to be properly formatted for small form-factor devices. They like to scan and discover content, often reading long form articles in an online or mobile environment, rather than waiting until they are back in the lab or library to do so. All of this information is clearly valuable, and while we can respond to and learn from these experiences and preferences, early research shows that this information by itself is not enough to develop our mobile strategy. Just as Dr. J. Murray Luck hit on a popular new concept for reducing the impact of the growing wealth of literature, our continuing task is to explore ideas and technologies beyond the expectations of our current readers to meet their future needs.

\section{Development is not just about technology}

Of course, finding success in the mobile environment is not just about the technological infrastructure required to deliver content effectively. We have also had to consider our business model, from what level of content to provide via mobile (we opted for full text) to whether and how to charge (users do not want to pay for content, and we decided to acknowledge this and avoid conflict with our library customers by enabling full text mobile access as part of an institutional subscription). Meanwhile, our focus groups also explored issues such as research paths and authority (Google, Google Scholar and Wikipedia were confirmed as common starting points for basic reference; editor and author affiliations were noted as important indicators of quality and legitimacy).

\section{Spread the risk, but integrate the strategy}

In the early stages of adapting to mobile, our strategy was one of experimentation. We were careful to spread our mobile investment across multiple projects to reduce the risk of total failure if any aspect didn't work as planned. Nonetheless, despite this inclusive approach, there were still choices and decisions to be made about the direction of our mobile activities. Should we create device-specific apps or focus on mobile websites? What about ebooks and formats such as ePub? Which devices should we support? Should we develop our mobile platform in house or outsource it to a technology partner? Which internal department should take the lead? In breaking down our mobile presence across multiple experiments, it was particularly important to coordinate our developmental decisions in an integrated strategy. As a non-profit organization, our focus was on meeting user demand and increasing usage of our content rather than directly generating new revenues. We wanted to be sure that our mobile activity did not cannibalize our core business and, as noted above, that it did protect the intermediary role of libraries.

Some clear principles governed our experimentation:

- Prioritize content over features: keep things simple, seamless and smart.

- We are a publisher, not a technology firm.

- Find new ways to connect users to articles ... but keep out of their way.

- Not all functionality needs to be replicated for mobile devices.

Our experimentation has included: 


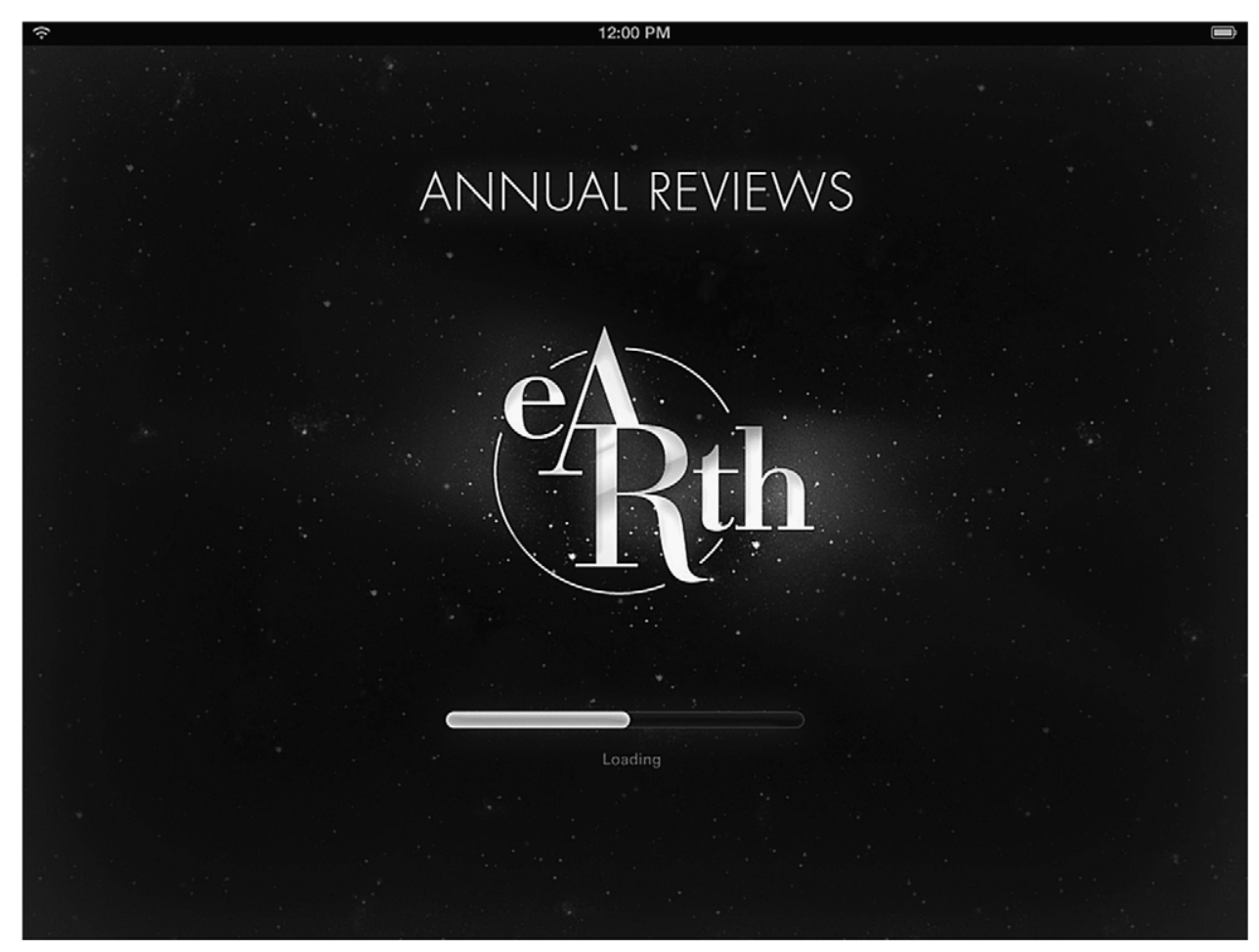

Fig. 2. Screenshot of Annual Reviews' eARth app.

- Developing a device-agnostic mobile website for full-text access to our journals.

- Converting our content into a range of different formats (ePub, Kindle, iBooks and "segmented" PDFs that avoid "multi-touch fatigue" by reflowing text).

- Experimenting with serendipitous discovery (and the "cool factor") in a visually rich browse-led "eARth app" for iPad (Fig. 2).

- Focusing on a specific audience with an app that tailors our content and functionality for economists (Fig. 3).

We have already suspended some ideas that clearly were not adding value in the way that we had hoped, for example, an offline "reader" app that was primarily structured around news and RSS feeds (rather than full text).

\section{Keep on evolving}

Our mobile journals site, built by our technology partner Atypon, initially required users to download a device-specific app to "pair" or connect their device with their institution to enable them to access their library's Annual Reviews subscriptions on the mobile website. Initial feedback showed users found the process lengthy and confusing, so in the next build we eliminated the device-specific app and simplified the authentication process. Now, institutional authentication takes place automatically and behind the scenes. Institutional authentication is key, as users experience a rather seamless connection to their library, and we are able to report usage of the mobile website within COUNTER statistics. (COUNTER 


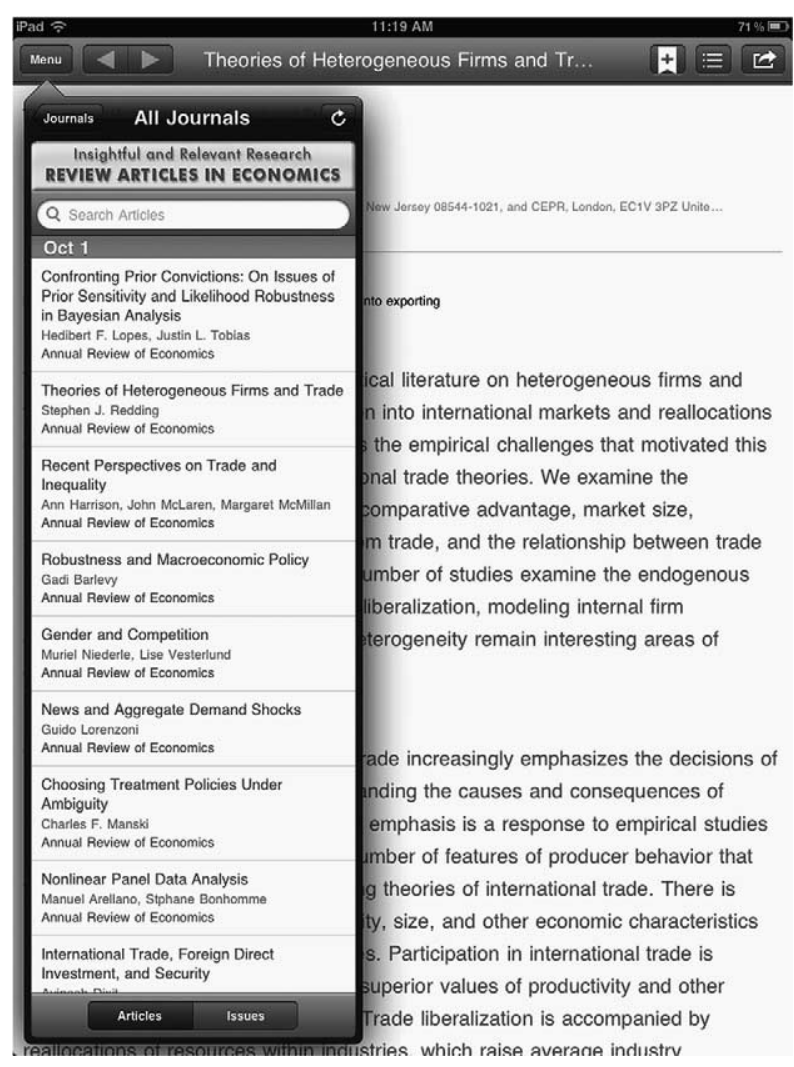

Fig. 3. Screenshot of "EconScholar" app.

reports are an important collection management tool for librarians and therefore part of our strategy to empower rather than disintermediate.)

\section{Lessons learned}

Beyond technical challenges, there are organizational challenges to embracing mobile. Internal stakeholders include technology, marketing, sales, editorial, production and customer service, and there may also be external partners such as developers and beta testers. At an early stage in the planning process, it is important to identify representatives from each group and manage their expectations and roles. Communication is essential; we found cross-functional team meetings valuable.

Meanwhile, mobile is no exception to the truism that innovation takes longer and costs more than anticipated. Plan to dedicate more time, money and resources than seem necessary upfront, and - given that experimentation should be broad - expect that some of your activities will never make it out of beta.

\section{Tips and advice}

(1) Be clear about your overall strategy, principles and desired outcomes and whether these are oriented toward usage, revenue or brand. 
(2) Know your core strength - what is the value you provide to your audience? - and do not lose sight of this as you experiment with mobile.

(3) Remember that mobile is a cultural revolution as well as a technological one - ensure that your decisions are consistent with wider organizational goals and relationships, and that you invest in getting the business aspects right.

(4) Undertake research to be sure that developments are based on an up-to-date understanding of your audience's information habits and preferences.

(5) Think beyond the results of the research about how your core value might be delivered in the future - users can only understand their needs in the context of current solutions, so you need not only to learn from them but also to lead them.

(6) Pursue different ideas, albeit focused initially on the most commonly used devices.

(7) Start with a complete and detailed functional specification for each project. Avoid false starts and "scope creep", i.e. do not let features, ideas and costs push beyond the boundaries of the initial project specification. Use expert partners to help identify difficulties and inconsistencies early on.

(8) Test early and often, and expect post-launch incremental updates to keep improving the user experience.

\section{Conclusion}

Annual Reviews has a long history of solving the problems that academics encounter. Our future role must continue to reflect our users' environment and workflows, without being entirely shaped by them. In my talk at NFAIS, I related our experience to the idea of "skeuomorphs" - derivative objects that retain design cues from original, defunct objects. There is value in basing new technology on old needs - users find it easier to adopt new tools if they can relate to a familiar process, function or design. Annual Reviews users depend on our review articles as a shortcut to access available literature, to fill in gaps in understanding of a topic or field, to cut out the "noise", and to connect to the research and ideas that really matter. In transitioning to mobile, we are balancing users' current, known needs with those of a future they cannot yet anticipate. To innovate effectively, we need to understand where our readers are now, and where they are going. Our essential task is to solve future problems before our users even realize they have them.

\section{Reference}

[1] J.M. Luck, Confessions of a biochemist, Annu. Rev. Biochem. 50 (1981), 1-23. 